\title{
Capacidad discriminativa del deterioro funcional del Inventario de Personalidad DMS-5 Short Form en pacientes con trastorno por uso de sustancias
}

\author{
Discriminative capacity for functional impairment \\ of the Personality Inventory for DSM-5 Short Form \\ in patients with substance use disorder
}

\begin{abstract}
Ana de la Rosa Cáceres*, Juan Ramírez López**, Fermín Fernández Calderón*,***, Oscar M. Lozano-Rojas****, Enrique Moraleda-Barreno****, Carmen Díaz-Batanero*,***.

* Departamento de Psicología Clínica y Experimental. Universidad de Huelva, Huelva, Spain; ** Servicio Provincial de Drogodependencias de Huelva, Huelva, Spain; *** Centro de Investigación en Recursos Naturales, Salud y Medio Ambitente (RENSMA). Universidad de Huelva, Huelva, Spain.
\end{abstract}

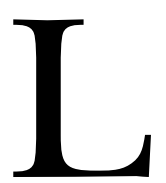
a literatura especializada muestra que los Trastornos de Personalidad (TP) presentan una alta comorbilidad con Trastorno por Uso de Sustancias (TUS). La mayor disfuncionalidad y peor respuesta terapéutica de pacientes comórbidos (Van Den Bosch y Verheul, 2007) apuntan la necesidad de evaluar la personalidad entre pacientes con TUS.

El Modelo Alternativo de Trastornos de Personalidad (MATP) propuesto en el DSM-5 propone la organización de los rasgos de personalidad de forma dimensional (Krueger y Markon, 2014). Uno de los instrumentos más empleados para evaluar este modelo es el Inventario de Personalidad DSM-5 (PID-5; Krueger, Derringer, Markon, Watson y Skodol, 2012). Se ha señalado necesario encontrar fórmulas que concilien las propuestas dimensionales con las decisiones categoriales de la práctica clínica (Alarcón, 2010). En este sentido, se han planteado puntos de cortes normativos del PID-5 que pretenden facilitar las decisiones clínicas (Gutiérrez et al., 2017; Samuel, Hopwood, Krueger, Thomas y Ruggero, 2013).

A pesar de los estudios que muestran relación entre las elevaciones en los rasgos y el desajuste funcional y psicosocial (Keeley, Flanagan y McCluskey, 2014), hasta la fecha, ningún estudio ha analizado la capacidad discriminativa del deterioro funcional del PID-5. Este trabajo analiza la sensibilidad y especificidad de cada una de las facetas para detectar deterioro funcional en un grupo de pacientes con
TUS. Además, se compara el uso de un criterio funcional y otro normativo para establecer los puntos de corte que representan un funcionamiento patológico en las facetas del MATP.

178 pacientes con TUS asistentes a centros de tratamiento ambulatorio de adicciones. Los hombres constituyeron el $82,6 \%$ de la muestra, con edad media de 41,28 años ( $D E$ $=11,24)$.

Se administró la versión en castellano del PID-5-Short Form (Díaz-Batanero, Ramírez-López, Domínguez-Salas, Fernández-Calderón y Lozano, 2019). La discapacidad funcional se evaluó con el cuestionario para la Evaluación de la Discapacidad de la Organización Mundial de la Salud (WHODAS 2.0; Üstün et al., 2010).

Los instrumentos fueron administrados por un psicólogo con experiencia en la evaluación de pacientes 15 días después del comienzo del tratamiento. Este estudio fue aprobado por el comité ético de la Universidad de Huelva.

Se estimaron las curvas ROC usando una puntuación total en la WHODAS $2.0>25$ como umbral para clasificar los pacientes con discapacidad moderada a extrema (Üstün et al., 2010). Se estimaron los puntos de corte, según el criterio funcional con el mejor balance entre sensibilidad y especificidad, fijando una especificidad mínima de ,70. Se compararon con los puntos de corte normativos, calculando las puntuaciones T > 65 (Gutiérrez et al., 2017).

Recibido: Junio 2019; Aceptado: Noviembre 2019.

Enviar correspondencia a: Carmen Díaz Batanero.

Dpto de Psicol. Clínica y Exper. Fac. de Educ., Psicol. y Cienc. de la Act. Física y Deporte. Campus del Carmen. Av. Fuerzas Armadas s/n, 21071 Huelva E-mail: carmen.diaz@dpsi.uhu.es / Tel. 959218428. Fax 959219201. 
El 35,4\% de la muestra mostraron discapacidad moderada o extrema. Los valores AUC oscilan entre ,503 (95\% $\mathrm{IC}=[, 41,59]$ ) (Búsqueda de atención) y ,787 (Depresión) $(95 \% \mathrm{IC}=[, 71,86])$ con un valor medio de ,657. Valores de AUC > ,7 fueron observados en: Anhedonia, Ansiedad, Depresión, Distractibilidad, Excentricidad, Irresponsabilidad, Perseveración y Sumisión. Sin embargo, seis facetas no muestran capacidad discriminativa: Búsqueda de atención, Grandiosidad, Evitación de la intimidad, Manipulación, Afectividad restringida y Perfeccionismo rígido. Los puntos de corte usando el criterio funcional son superiores en las todas las facetas que resultan discriminativas del deterioro funcional respecto a los obtenidos según criterios normativos (excepto Sumisión y Asunción de riesgos).

Tabla 1. Resultados de los análisis ROC y puntos de corte estimados a través de los criterios normativos y de discapacidad funcional.

\begin{tabular}{|c|c|c|c|c|c|c|c|c|}
\hline & AUC [C195\%] & $p$ & $\begin{array}{l}\text { Criterio } \\
\text { funcional }\end{array}$ & Sensibilidad & Especificidad & Criterio normativo & Sensibilidad & Especificidad \\
\hline Anhedonia &, $744[, 66-, 82]$ & $<, 001$ & 1,87 & ,710 & ,722 & 1,25 & ,823 &, 583 \\
\hline Ansiedad & ,770[,70 - ,84] & $<, 001$ & 2,37 & ,613 & ,765 & 1,78 & ,806 &, 574 \\
\hline Búsqueda de at. &, $503[, 41-, 59]$ & ,949 & 0,87 & ,403 &, 722 & 1,49 & ,145 & ,835 \\
\hline Insensibilidad &, $595[, 50-, 68]$ &, 036 & 0,62 &, 436 & ,748 & 0,64 & ,436 & ,748 \\
\hline Depresión & ,787 [,71 - ,86] & $<, 001$ & 1,12 &, 677 & ,765 & 0,95 &, 742 & ,696 \\
\hline Distractibilidad & ,777 [,70 - , 84] & $<, 001$ & 2,12 &, 677 & ,757 & 1,54 & ,855 &, 574 \\
\hline Excentricidad & ,760[,68 - , 83] & $<, 001$ & 1,62 &, 678 & ,730 & 1,33 & ,726 & ,591 \\
\hline Labilidad emocional & ,648[,56 - ,73] & ,001 & 2,12 &, 516 &, 722 & 2,03 &, 516 &, 722 \\
\hline Grandiosidad &, $557[, 46-, 64]$ & ,209 & 0,87 & ,290 & ,782 & 1,24 & ,177 & ,896 \\
\hline Impulsividad &, $642[, 55-, 72]$ &, 002 & 2,12 & ,403 & ,730 & 1,60 & ,758 & ,609 \\
\hline Evit. de la intimidad &, $585[, 49-, 67]$ & ,078 & 1,87 & ,387 &, 765 & 1,15 & ,468 &, 643 \\
\hline Irresponsabilidad & ,702[,62 - ,78] & $<, 001$ & 1,37 &, 532 & ,801 & 0,92 & ,774 & ,487 \\
\hline Manipulación &, $574[, 48-, 66]$ & ,105 & 0,87 & ,355 &, 735 & 1,27 &, 226 & ,878 \\
\hline Disreg. Perceptiva &, $635[, 54-, 72]$ &, 003 & 0,87 & ,403 & ,774 & 0,87 & ,403 &, 774 \\
\hline Perseveración & ,713 [,63 - ,79] & ,001 & 1,87 &, 565 & ,747 & 1,54 &, 662 & ,661 \\
\hline Afectividad restringida &, $543[, 45-, 63]$ & ,346 & 1,62 & ,355 &, 725 & 1,41 &, 516 & ,614 \\
\hline Perfeccionismo ríg. &, $574[, 48-, 66]$ & ,106 & 1,62 & ,339 & ,713 & 1,82 & ,290 & ,791 \\
\hline Asunción de riesgos &, $631[, 54-, 72]$ & ,004 & 1,12 &, 532 & ,703 & 1,65 & ,323 & ,896 \\
\hline Ins. por separación & $677[, 59-, 75]$ & $<, 001$ & 2,12 & ,435 &, 774 & 1,61 &, 677 &, 643 \\
\hline Sumisión & ,703 [,62 - ,78] & $<, 001$ & 1,12 &, 548 & ,765 & 1,47 & ,468 & ,861 \\
\hline $\begin{array}{l}\text { Creencias y experiencias } \\
\text { inusuales }\end{array}$ & $639[, 55-, 72]$ & ,002 & 1,62 & ,3556 &, 783 & 1,03 &, 581 &, 574 \\
\hline Retraimiento & ,684[,60 - ,76] & $<, 001$ & 1,62 &, 532 & ,735 & 1,23 & ,597 & ,835 \\
\hline
\end{tabular}

Los resultados muestran que el PID-5 tiene buena capacidad discriminativa de la disfuncionalidad evaluada por el WHODAS 2.0 en la mayoría de facetas. Estudios previos han mostrado esta relación, particularmente en las dimensiones Comprensión y comunicación, Relaciones y Participación en sociedad (Díaz-Batanero et al., 2019; Keeley et al., 2014). Se observa mayor capacidad discriminativa en las facetas vinculadas a la afectividad negativa, dimensión asociada a mayores niveles de patología y disfuncionalidad (Watson, Stasik, Ro y Clark, 2013). Por el contrario, las facetas Búsqueda de atención, Insensibilidad, Grandiosidad, Hostilidad, Impulsividad, Evitación de la Intimidad, Mani- pulación, Afectividad restringida y Perfeccionismo rígido no presentan capacidad discriminativa. Congruentemente, trabajos previos encontraron que Búsqueda de atención, Grandiosidad, Afectividad restringida, Evitación de la intimidad y Perfeccionismo rígido mostraban menores diferencias entre muestras clínicas y comunitarias (Gutiérrez et al., 2017).

17 de las 25 facetas muestran puntos de corte usando criterio funcional superiores a los obtenidos con criterios normativos y racionales (Samuel et al., 2013). Globalmente, se podría sugerir que el uso de criterios normativos sería más adecuado para estudios epidemiológicos poblacio- 
nales. Sin embargo, los criterios funcionales podrían ser más útiles en muestras clínicas, permitiendo a los terapeutas planificar tratamientos más específicos para los trastornos que causan una mayor discapacidad funcional de los pacientes.

La evaluación del desajuste funcional exclusivamente con auto-informes puede suponer una limitación. Un uso óptimo de múltiples fuentes de datos podría mejorar la predicción del comportamiento en la evaluación psicopatológica y funcional. Estudios futuros deberían complementar los datos obtenidos con información provista por otros informantes cercanos.

\section{Reconocimientos}

Este estudio ha sido financiado por el proyecto "Estudio longitudinal sobre el efecto del tratamiento en la recuperación de la función ejecutiva en pacientes con adicción al alcohol y cocaína: implicaciones sobre los resultados del tratamiento" del Plan Nacional sobre Drogas (Q7150008F-2016/034).

\section{Conflicto de intereses}

No existe conflicto de intereses.

\section{Referencias}

Alarcón, R. D. (2010). Hacia nuevos sistemas de diagnóstico: proceso, preguntas y dilemas. Revista de Psiquiatría y Salud Mental, 3, 37-39. doi:10.1016/j.rpsm.2009.07.001.

Díaz-Batanero, C., Ramírez-López, J., Domínguez-Salas, S., Fernández-Calderón, F. y Lozano, Ó. M. (2019). Personality inventory for DSM-5-short form (PID-5-SF): Reliability, factorial structure, and relationship with functional impairment in dual diagnosis patients. Assessment, 26, 853-866.

Gutiérrez, F., Aluja, A., Peri, J. M., Calvo, N., Ferrer, M., Baillés, E.,... Krueger, R. F. (2017). Psychometric properties of the Spanish PID-5 in a clinical and a community sample. Assessment, 24, 326-336. doi:10.1177/1073191115606518.

Keeley, J. W., Flanagan, E. H. y McCluskey, D. L. (2014). Functional impairment and the DSM-5 dimensional system for personality disorder. Journal of Personality Disorders, 28, 657-674. doi:10.1521/pedi_2014_28_133.

Krueger, R. F. y Markon, K. E. (2014). The role of the DSM-5 Personality Trait Model in moving toward a quantitative and empirically based approach to classifying personality and psychopathology. Annual Review of Clinical Psychology, 10, 477-501. doi:10.1146/annurev-clinpsy-032813-153732.

Krueger, R. F., Derringer, J., Markon, K. E., Watson, D. y Skodol, A. E. (2012). Initial construction of a mala- daptive personality trait model and inventory for DSM5. Psychological Medicine, 42, 1879-1890. doi:10.1017/ S0033291711002674.

Samuel, D. B., Hopwood, C. J., Krueger, R. F., Thomas, K. M. y Ruggero, C. J. (2013). Comparing methods for scoring personality disorder types using maladaptive traits in DSM-5. Assessment, 20, 353-361. doi:10.1177/1073191113486182.

Üstün, T. B., Chatterji, S., Kostanjsek, N., Rehm, J., Kennedy, C., Epping-Jordan, J.,... Pull, C. (2010). Developing the World Health Organization Disability Assessment Schedule 2.0. Bulletin of the World Health Organization, 88, 815-823. doi:10.2471/blt.09.067231.

Van Den Bosch, L. M. y Verheul, R. (2007). Patients with addiction and personality disorder: Treatment outcomes and clinical implications. Current Opinion in Psychiatry, 20, 67-71. doi:10.1097/YCO.0b013e328011740c.

Watson, D., Stasik, S. M., Ro, E. y Clark, L. A. (2013). Integrating normal and pathological personality: Relating the DSM-5 trait-dimensional model to general traits of personality. Assessment, 20, 312-326. doi:10.1177/1073191113485810. 